\title{
Dynamic optimization of a 2-DOF pseudo-equatorial tracking system in virtual prototyping concept
}

\author{
C. Alexandru, M. Comşiț, P. Alexandru \\ Product Design and Robotics Department \\ Centre "Product Design for Sustainable Development" \\ University "Transilvania" of Braşov \\ 29 Bd. Eroilor, 500036 Braşov (Romania) \\ Phone/Fax:+40268472496, e-mail: calex@unitbv.ro, comsit@unitbv.ro, alex.p@unitbv.ro
}

\begin{abstract}
The key word for the design process of the photovoltaic tracking systems is the energetic efficiency. Using the tracking system, the photovoltaic panel follows the sun and increase the collected energy, but the driving motors \& actuators consume a part of this energy. In these terms, the optimization of the tracking system became an important provocation in the modern research and technology.
\end{abstract}

In this paper, we evaluate a strategy for the dynamic optimization of the photovoltaic tracking systems. The main task in optimization is to maximize the energetic gain by increasing the incoming solar radiation and minimizing the energy consumption for tracking. This strategy is possible by developing the virtual prototype of the tracking system, which is a control loop composed by the multibody mechanical model connected with the dynamic model of the actuators and with the controller model. In this way, we are able to optimize the tracking mechanism, choose the appropriate actuators, and design the optimal controller.

\section{Key words}

Photovoltaic panel, optimization, tracking mechanism, control system, virtual prototype, energy balance.

\section{Introduction}

Solar energy conversion is one of the most addressed topics in the field of renewable energy systems. The technical solution for converting the solar energy in electricity is well-known: the photovoltaic systems. The energetic efficiency of the photovoltaic systems depends on the degree of use and conversion of the solar radiation. There are two ways for maximizing the rate of useful energy: optimizing the conversion to the absorber level, and increasing the incident radiation rate by using mechanical tracking systems [1].

Generally, the tracking systems contain mechanisms (e.g. linkages, gear mechanisms, cam mechanisms, chain or belt transmissions), which are driven by controlled rotary motors and/or linear actuators. The orientation of the photovoltaic panels may increase the efficiency of the conversion system from $20 \%$ up to $50 \%$ [2]-[4].
The key word for the design process of the tracking systems is the energetic efficiency; using the tracking system, the photovoltaic panel follows the sun and increase the collected energy, but the driving motors consume a part of this energy. The tracking system is efficient if the difference among the energy produced by the photovoltaic panel with tracking system and the same panel without tracking (fixed) is larger than the energy consumption for orientation, as follows:

$$
\varepsilon=\left(E_{P T}-E_{P F}\right)-E_{C}>>0,
$$

where $\mathrm{E}_{\mathrm{PT}}$ is the electric energy produced by the photovoltaic panel with tracking, $E_{\mathrm{PF}}$ - the energy produced by the same panel without tracking (fixed), and $\mathrm{E}_{\mathrm{C}}$ - the energy consumption for orienting the panel. In the current conditions, the maximization of the parameter $\varepsilon$, by optimization of the tracking system, became an important challenge in the modern research.

Determining the real behaviour of the tracking system is a priority in the design stage since the emergence of the computer graphic simulation. The research of the literature reveals the limits of the actual stage in the development of the tracking mechanisms (i.e. the mechanical device). The literature presents some constructive solutions of tracking mechanisms [3]-[6], but since now there are no unitary modelings on structural, kinematical and dynamical aspects in designing the tracking mechanisms. The necessity of a unitary analysis \& optimization method can be assured by using the Multi Body Systems (MBS) theory, which facilitates the self-formulating algorithms, having as main goal the reducing of the processing time, for making possible the real-time simulation [7],[8].

In the design process of the tracking systems, the solar radiation represents the main input data. Interacting with atmospheric phenomena involving reflection, scattering, and absorption of radiation, the quantity of solar energy that reaches the earth's surface is reduced in intensity. The total solar radiation received at ground level includes two main components: direct solar radiation and diffuse radiation [9]. 
The solar radiation can be measured using traditional instruments, or can be digitally recorded with a data acquisition system. In addition, different models were developed for estimating the solar radiation. The traditional Angstrom's linear approach is based on measurements of sunshine duration, while relatively new methods are based on artificial neural networks - ANN [10]. Different models for estimating the monthly mean solar radiation, including linear Angstrom - Prescot variation, quadratic equation, logarithmic variation, and exponential function have been studied in [11]; the root mean square error is the principal elements of this comparative analysis.

Other papers in literature refer to the computation of the yearly energy collection allowed by different tracking strategies. Using as input data the location latitude and commonly available values of monthly irradiation, a relation between the latitude of the chose location and the most suitable solar tracker is established [12]. An analysis model for comparing the energy capture between fixed tilt angle and sun tracking systems, in clear sky and mean sky conditions, has been developed in [3]. Specific software tools were realized to simulate the energy yield of PV systems as a function of the ground cover ratio, for different tracking strategies [13].

Another subject in literature is the control strategy of the photovoltaic systems with tracking. Usually, closed loop systems with photo sensors are used. The photo sensors are responsible for discrimination of the sun position and for sending electrical signals, proportional with the error, to the controller, which actuates the motors to track the sun. Many authors have adopted this method as a basis in construction and design of such systems [6, 14]. Although, the orientation based on the sun detecting sensors, may introduce errors in detection of real sun position for variable weather conditions.

The opened loop systems are based on mathematic algorithms that may provide predefined parameters for the motors, depending on the sun positions on the sky dome. These positions can be precisely determined because they are functions of the solar angles that can be calculated for any local area [2]. By using this control technique, based on predefined parameters, the errors introduced by the use of the sensors may be avoided. Other strategy is to develop a hybrid control systems that consists of a combination of opened loop tracking strategies based on solar movement models and closed loop strategies using a dynamic feedback controller [15].

From the controller point of view, different strategies are used such as classical techniques as PID algorithm, or more advanced strategy such as fuzzy logic controller FLC [2],[5]. The implementation of a fuzzy logic neural controller, which is an evolution of the fuzzy control concept, allows the photovoltaic system to learn control rules [16].

In the above-presented conditions, our contributions can be structured in the following directions: developing a general method, based on the MBS theory, for the structural synthesis of the tracking systems; developing a mathematic model for establishing the incident solar radiation, depending on the angle of incidence; developing the analysis \& optimization flow-chart of the tracking systems; developing the virtual prototyping platform for simulating the tracking systems in real operating conditions; developing the control system and integrating the control in the mechanical model of the tracking system at the virtual prototype level; evaluating the energetic efficiency of the tracking systems. The application in this paper is made for a pseudo-equatorial dual-axis tracking system, at which the daily and the seasonal motions are performed with linear actuators.

\section{Developing the Solution}

The orientation principle of the photovoltaic panels is based on the input data referring to the position of the sun on the sky dome. For the highest conversion efficiency, the sunrays have to fall normal on the receiver so the system must periodically modify its position in order to maintain this relation between the sunrays and the panel. The positions of the Sun on its path along the year represent input data for the design process of the tracking systems. The Earth describes along the year a rotational motion following an elliptical path around the sun. During one day, the Earth also spins around its own axis describing a complete rotation that generates the sunrises and the sunsets. The variation of the altitude of the sun on the celestial sphere during one year is determined by the precession motion, responsible for a declination of the Earth axis in consideration with the plane of the elliptic yearly path. Thus, for the design process of the tracking systems there are considered two rotational motions: the daily motion, and the yearly precession motion.

Consequently, there are two basic types of tracking systems: single-axis tracking systems, and dual-axis tracing systems. The single-axis tracking systems spins on their axis to track the sun, facing east in the morning and west in the afternoon. The tilt angle of this axis equals the latitude angle of the loco because this axis has to be always parallel with the polar axis. In consequence for this type of tracking system is necessary a seasonal tilt angle adjustment.

The two-axis tracking systems combine two motions, so that they are able to follow very precisely the sun path along the period of one year. Depending on the relative position of the revolute axes, there are three types of dual-axis systems: azimuthal, equatorial or polar, and pseudo-equatorial. For the azimuthal trackers, the daily motion is made by rotating the panel around the vertical axis, so that it is necessary to continuously combine the vertical rotation with an elevation motion around the horizontal axis. For the equatorial (polar) tracking systems, there are two independent motions, because the daily motion is made rotating the panel around the polar axis. The pseudo-equatorial tracking systems have an axis parallel with the revolute axis that determines the seasonal variation of the sun position on the sky dome; the revolute axis for the daily orientation oscillates relative to the seasonal axis. 
In order to identify suitable tracking systems, the structural synthesis has been performed by using the Multi-Body Systems (MBS) theory, according to which a mechanical system is defined as a collection of bodies with large translational and rotational motions, linked by simple or composite joints. The structural design consists in the following stages: identifying all possible graphs, taking into account the space motion of the system, the type of joints, the number of bodies, and the degree of mobility of the multibody system; selecting the graphs that are admitting supplementary conditions imposed by the specific utilization field; transforming the selected graphs into mechanisms by mentioning the fixed body and the function of the other bodies, identifying the distinct graphs versions based on the preceding particularizations, transforming these graphs versions into mechanisms by mentioning the types of geometric constraints (ex. revolute joint - R, or translational joint $\mathrm{T})$. The graphs of the multibody system are defined as features based on the modules, considering the number of bodies and the relationships between them (ex. R, T, RR, R-T, RR-RR, RR-RT and so on).

In the structural synthesis, there can be taken considered general criteria, for example the degree of mobility of the mechanism $(\mathrm{M}=1$ for the single-axis tracking systems, and $\mathrm{M}=2$ for the dual-axis tracking systems), the number of bodies, and the motion space/spatiality $(\mathrm{S}=3$ in the planar space, and $S=6$ in the general spatial case), as well as specific criteria, for example the type of the joint between the base and the input and/or output body. In this way, the structural synthesis method was applied and a collection of possible structural schemes of tracking mechanisms were obtained [17]. Some representative structural solutions will be implemented in "The Centre Product Design for Sustainable Development" from Transilvania University of Braşov, fact that will provide us a good opportunity to have a pertinent comparison between the real system performances and the results of the dynamic analysis developed on the virtual prototype.

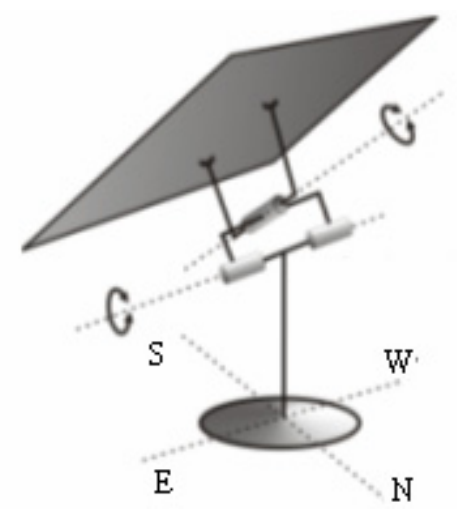

Fig. 1. The model of the pseudo-equatorial tracking system.

In this paper, the application is made for a pseudoequatorial tracking system, whose simplified model is shown in figure 1 , which is able to follow very precisely the sun path along the period of one year. The both daily and elevation motions are performed using linear actuators (fig. 2). The panel is mounted on a support, which rotates around a horizontal axis for generating the elevation. The daily motion is made by rotating the panel relative to the support. The linear actuators act between support and panel (for the daily motion), respectively between pillar and support (for elevation). The solution for system used in our study was selected from the multitude of the structural solution by using of the Multi Criteria Analysis. The evaluation criteria of the solutions were referring to the tracking precision, the amplitude of the motion, the complexity of the system, design and easy manufacturing and implementation.

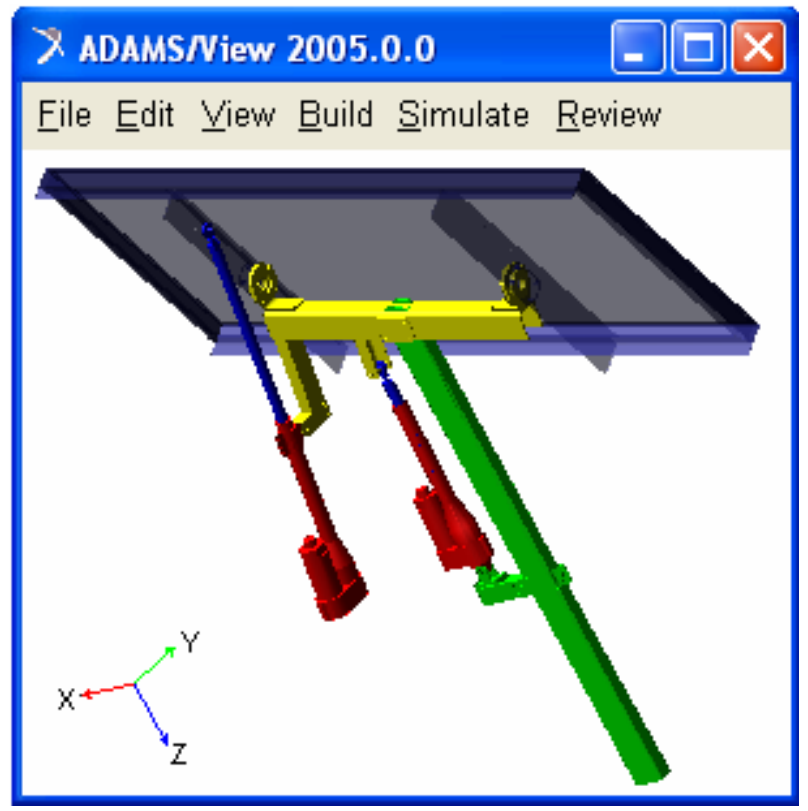

Fig. 2. The virtual prototype of the tracking system.

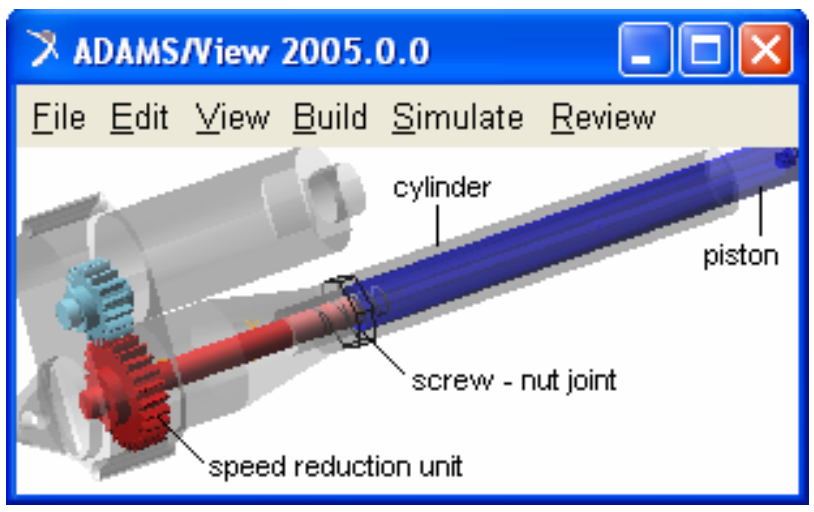

Fig. 3. The detailed model of the actuator.

The dynamic model of the tracking system includes the actuating motors, the bodies (with mass \& inertia properties), the geometric constraints between parts (i.e. the joints), and the external and internal forces \& torques. The model can be completed with other external factors, for example the wind or snow action. The revolute motion developed by motors is transformed in linear motion between the actuator components (cylinder and piston) by using screw - nut mechanisms (fig. 3), which also assure the irreversibility of the motion, necessary for blocking the system in the stationary positions. The geometry and the characteristics of the conversion system correspond to a Vitovolt 200 photovoltaic panel (active surface $-1.26 \mathrm{~m}^{2}$, weight $-15.5 \mathrm{Kg}$ ). 
The study has imposed the development of the virtual prototype of the tracking system (see fig. 2), which is a control loop composed by the multibody mechanical model connected with the dynamic model of the actuators and with the controller. For realizing this model, we used a virtual prototyping platform that includes CAD, MBS and C\&C software solutions. The CAD software (CATIA) was used to create the solid model of the tracking system, which contains information about the mass \& inertia properties of the bodies. The MBS software (ADAMS/View) was used for analyzing, optimizing and simulating the dynamic model of the photovoltaic tracking system. Using the C\&C software (MATLAB/Simulink \& ADAMS/Controls), the control system of the solar tracker was developed, in concurrent engineering concept, by integrating the control system model and the mechanical system model at the virtual prototype level. In this way, the physical testing process is greatly simplified, and the risk of the control law being poorly matched to the real system is eliminated.

The purpose of the dynamic model is to allow the evaluation of the tracking system behavior in real operating condition. Having in view to evaluate the energetic efficiency of the tracking system, the main parameters that are taken into consideration in the dynamic study are the motor torques for driving the system. The aim of the optimization is to minimize these parameters, in fact the energy consumption for moving the photovoltaic system. In our philosophy, this objective is performed by optimizing the motion law of the panel, for minimizing the energy consumption $\left(\mathrm{E}_{\mathrm{C}}\right)$, and maximizing the radiation absorption $\left(\mathrm{E}_{\mathrm{PT}}\right)$.

\section{The Control System}

For simulating the real behavior of the tracking system, in order to obtain more realistic results, we have developed the control system in the concurrent engineering concept, using ADAMS/Controls and MATLAB/Simulink. For connecting the mechanical model and the control system, the input \& output parameters have been defined. The control torques developed by the driving motors represents the input parameters in the mechanical model. The outputs, which are transmitted to the controller, are the daily and elevation angles of the photovoltaic panel.

For the input state variables, the run-time functions are 0.0 during each step of the simulation, because the control torques will get their values from the control system. The run-time functions for the input variables are defined using a specific ADAMS function - VARVAL, which returns the value of the given. For the output state variables, the run-time functions return the angles about the revolution axes: daily_ angle - AZ(panel.MAR_1, support.MAR_2), which returns the rotational displacement of one coordinate system marker attached to panel about the Z-axis of another marker attached to support; elevation_angle - AX(support.MAR_3, base.MAR_4), which returns the rotation of one marker attached to support about the $\mathrm{X}$-axis of another marker attached to base.
The next step is to export the ADAMS plant files for the control application. The input and output information are saved in a specific file for MATLAB (*.m). ADAMS also generates a command file (*.cmd) and a dataset file (*.adm) that are used during simulation. With these files, the control system block was created in MATLAB/ Simulink (fig. 4). In this model, beta.mat, gama.mat are the databases of the daily and elevation angles, which are explained in the next section of the paper. The mechanical system block (adams_sub) includes the MSC.ADAMS Plant. In this block there are configured the solver type, the user executable, the model file prefix, the output files prefix, the communication interval, the number of communications per output step, the interpolation order, and the simulation mode.

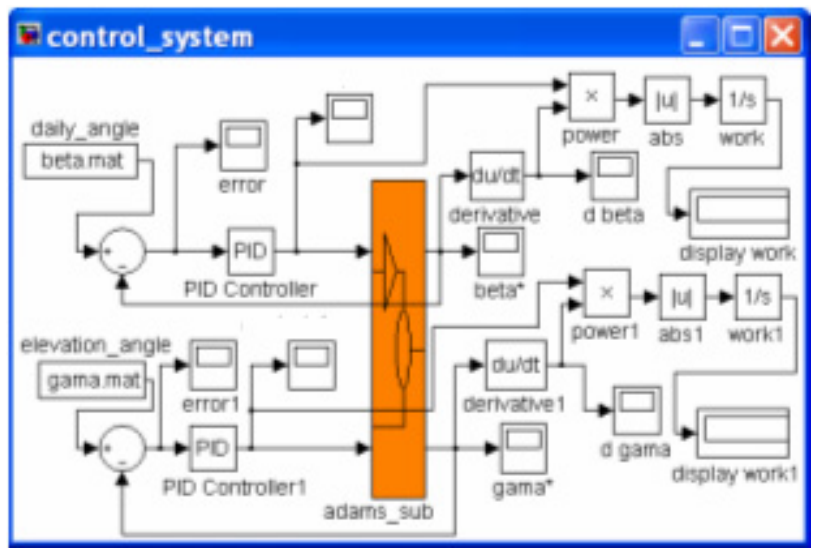

Fig. 4. The control system block.

From the controller point of view, for obtaining reduced transitory period and small errors, we used two PID controllers, one for each degree of freedom. The specific parameters of the PID controllers have been established having in view the following conditions: the increasing of the proportional term generates the decreasing of the transitory period from the dynamic response of the system, and of the position error, respectively; the integral term generates a class of dynamic responses and attenuates the error history; the derivative term generates a class of dynamic responses and amortizes the error.

In this mechatronic model, ADAMS accepts the control torques from MATLAB and integrates the mechanical model in response to them. At the same time, ADAMS provides the current daily and elevation angles for MATLAB to integrate the control system model. This model was used for the dynamic optimization of the tracking system; this subject is presented in the next section of the paper.

\section{The Optimization Procedure}

The photovoltaic panel can be rotated without brakes during the day-light, or can be discontinuously driven (step-by-step motion), usually by rotating the panel with equal steps at every hour. Obviously, the maximum incident solar radiation is obtained for the continuous motion, but in this case the operating time of the motors (consequently, the energy consumption) is high. The key 
idea is to maximize the energy gained through the stepby-step orientation, for absorbing a quantity of solar energy closed by the ideal case (continuous orientation), and to minimize the energy consumption for realizing this in-steps orientation. The optimization is made by reducing the angular field of the axes and the number of steps (the operating time of the motors), without significantly affecting the incoming solar energy.

The energy produced by the photovoltaic panel depends on the quantity of incident radiation, as well as the panel efficiency. The incident radiation, which is normal to panel, is given by the relation [1],[9]:

$$
G_{I}=G_{D} \cdot \cos i,
$$

where $G_{D}$ is the direct terrestrial radiation, and $\mathrm{i}$ - the angle of incidence. The direct radiation is established using the next equation [9]:

$$
G_{D}=G_{0} \exp \left(-\frac{T_{R}}{0.9+9.4 \cdot \sin \alpha}\right)
$$

in which:

$$
\begin{gathered}
G_{0}=\bar{G}_{0} \cdot(1+0.0334 \cdot \cos x), \\
x=0.9856^{\circ} \cdot n-2.72^{\circ}, \\
\alpha=\sin ^{-1}(\sin \delta \cdot \sin \varphi+\cos \delta \cdot \cos \varphi \cdot \cos \omega), \\
\omega=15^{\circ} \cdot(12-T),
\end{gathered}
$$

where $\mathrm{G}_{0}$ is the extraterrestrial radiation, $\overline{\mathrm{G}}_{0}$ - the solar constant $\left(1367 \mathrm{~W} / \mathrm{m}^{2}\right), \mathrm{n}$ - the day number during a year, $\mathrm{T}_{\mathrm{R}}$ - the distortion factor, which depends on the month and the geographic region, $\alpha$ - the solar altitude angle, $\delta$ the solar declination, $\varphi$ - the location latitude, $\omega$ - the solar hour angle, and $\mathrm{T}$ - the local solar time.

The angle of incidence is determined from the scalar product of the sun's ray vector and the normal vector on panel [18], as follows:

$$
i=\cos ^{-1}\left(\cos \beta \cdot \cos \beta^{*} \cdot \cos \left(\gamma-\gamma^{*}\right)+\sin \beta \cdot \sin \beta^{*}\right),
$$

in which:

$$
\begin{gathered}
\beta=\sin ^{-1}(\cos \delta \cdot \sin \omega), \\
\gamma=\sin ^{-1}\left(\frac{\cos \alpha \cdot \cos \psi}{\cos \beta}\right), \\
\psi=(\sin \omega) \cos ^{-1}\left(\frac{\sin \alpha \cdot \sin \varphi-\sin \delta}{\cos \alpha \cdot \cos \varphi}\right),
\end{gathered}
$$

where $\beta$ and $\gamma$ are the diurnal $\&$ seasonal angles of the sun's rays in the pseudo-equatorial system, $\beta^{*}$ and $\gamma^{*}$ the daily \& elevation angles of the panel (theoretically, $\gamma^{*}=\varphi-\delta$ ), and the $\psi-$ the azimuth angle.
With this mathematic algorithm, we are able to estimate the incident radiation in every day during a year, for different locations and tracking strategies. It has been demonstrated that for every month there is one day whose irradiation is equal to the monthly average: it is the day in which the declination equals the mean declination of the month [12]. Due to this consideration, a noticeable facilitation is introduced in the computing calculation, considering just the mean days of each month instead of the 365 days of the year. Our paper presents the exemplification for the equinox path of the sun. The situation is similar for any period of the year: the energy gain is not affected by the variation of the radiation intensity because the gain is obtained from the difference between a tracked panel energy output and a non-tracked panel energy output. The numeric simulations were performed considering the Braşov area, with the following input data: $\varphi=45.5^{\circ}, \delta=23.45^{\circ}, n=172$, $\mathrm{T} \in[4.26,19.74]$, and $\mathrm{T}_{\mathrm{R}}=4.2$.

\section{Results and Conclusions}

Intending to minimize the energy consumption, we have adopted a solution in which the orientation is made in steps. Such tracking strategy was formulated in [18], using optimal algorithms based on the number of steps necessary for orientation. The algorithms are developed considering the correlation between the maximum amplitudes of the motion and the number of the tracking steps. Thus, we obtained the optimal variations of the daily \& elevation angles of the panel, with the following fields (fig. 5): $\beta^{*} \in\left[-60^{\circ},+60^{\circ}\right], \gamma^{*} \in\left[11^{\circ}, 22.05^{\circ}\right]$. The operating time for a step is $0.1 \mathrm{~h}$ (for the both motions).

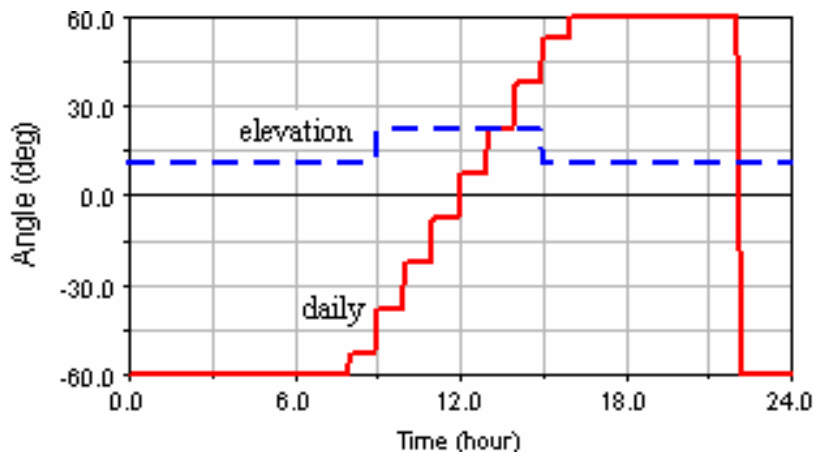

Fig. 5. The variation of the daily and elevation angles.

With these motions, we have obtained the variation of the incident radiation during the summer solstice day, which is shown in figure 6 (curve a). In the same figure, there are presented the incident radiation obtained for the fixed panel (b), in the solar noon position $\left(\beta^{*}=0^{\circ}\right.$, and $\gamma^{*}=$ $22.05^{\circ}$ ), and the maximum radiation that can be obtained through the continuous orientation, when the angle of incidence is null for the entire light-day period (c). Considering the active surface and the panel's efficiency, we have obtained the energy (mechanical work) produced by the panel, as follows: panel with step-bystep orientation (see fig. 5) - $\mathrm{E}_{\mathrm{PO}}=1743 \mathrm{~J}$; fixed panel $\mathrm{E}_{\mathrm{PF}}=1224 \mathrm{~J}$; panel with continuous orientation - $\mathrm{E}_{\mathrm{PO} \text { max }}$ $=1783 \mathrm{~J}$. As we can see, the step by step tracking generates a quantity of energy closed-by the ideal case. 


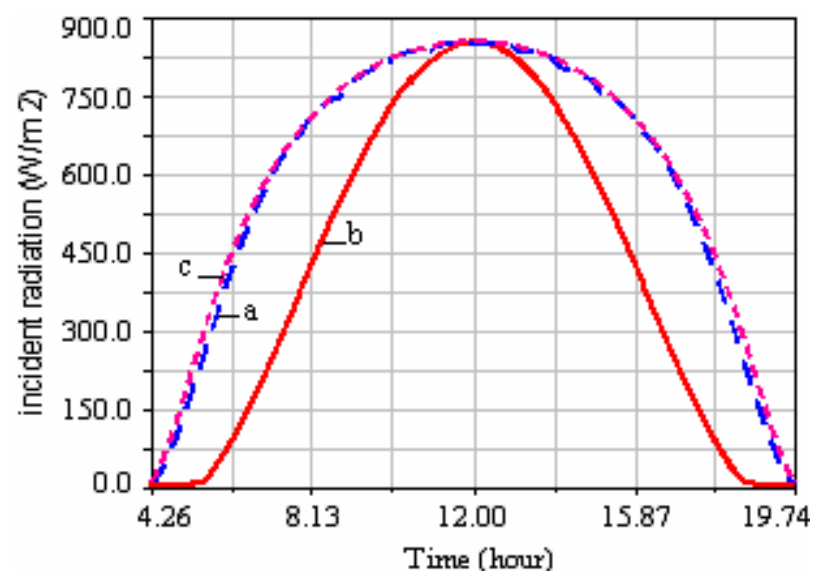

Fig. 6. The variation of the incident radiation.

On the other hand, using the mechatronic model described in the previous sections, we are able to compute the energy consumption for realizing the tracking law, which depends on the control torques generated by the driving motors, as well as the angular velocities of the input elements. For the step by step tracking, the energy consumption curves are shown in figure 7 (a - daily motion, b - elevation, c - total consumption). With these values, the efficiency coefficient of the tracking system has been computed (see rel. 1 ), $\varepsilon=483$, which means an energy contribution of $39.5 \%$ relative to the fixed panel. This value confirms the viability of the "in-steps" optimization strategy.

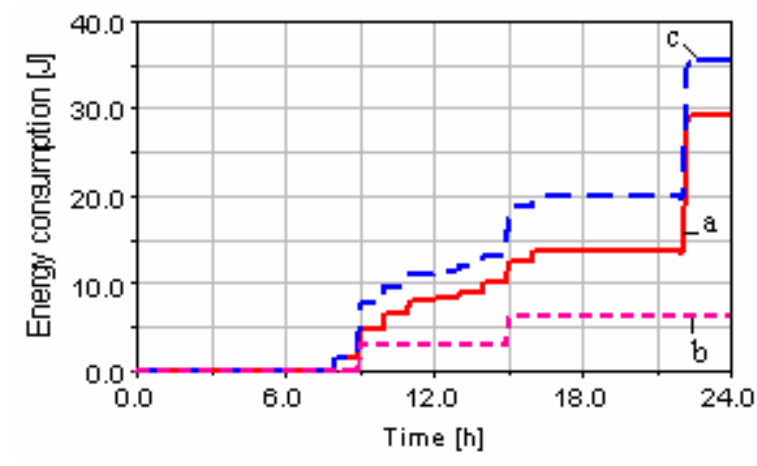

Fig. 7. The energy consumption for orientation.

The application is a relevant example regarding the implementation of the virtual prototyping tools in the design process of the tracking systems. One of the most important advantages of this kind of simulation is the possibility to perform virtual measurements in any point or area of the tracking system, and for any parameter. The optimization procedure leads to an efficient tracking system, without developing expensive hardware prototypes. In this way, the behavioral performance predictions are obtained much earlier in the design cycle of the tracking systems, thereby allowing more effective and cost efficient design changes.

The tracking system proposed will be manufactured and implemented, creating a real perspective for the research in the field. This allows a relevant comparison between the virtual prototype analysis and the concrete data achieved by measurements.

\section{Acknowledgement}

The Romanian Ministry of Education financially supported this research in the frame of the CNCSIS project, code 892/2007.

\section{References}

[1] Y. Goswami, F. Kreith and J. Kreder, Principles of solar engineering, Taylor \& Francis, Philadelphia (1999).

[2] S. Abdallah and S. Nijmeh, "Two-axis sun tracking with PLC control", Energy Conversion and Management, Vol. 45, No. 12, pp. 1931-1939, 2004.

[3] A. Canova, L. Giaccone and F. Spertino, "Sun tracking for capture improvement", in Proc. EUPVSEC'2007, Milano, pp. 3053-3058.

[4] H.D. Mohring, F. Klotz and H. Gabler, "Energy yield of PV tracking systems", in Proc. EUPVSEC'2006, Dresden, pp. 2691-2694.

[5] S. Odeh, "Design of a single-axis tracking collector", in Proc. EUROSUN'2004, Freiburg, pp. 527-532.

[6] P. Roth, A. Georgiev and H. Boudinov, "Design and construction of a sun-tracking system", Renewable Energy, Vol. 29, No. 3, pp. 393-402, 2004.

[7] J. Garcia de Jalón and E. Bayo, Kinematic and dynamic simulation of multibody systems, Springer Verlag, New York (1994).

[8] W. Schiehlen, "Multibody systems dynamics", Multibody Systems Dynamics, Vol. 1, No. 2, pp. 149-188, 1997.

[9] M. Meliß, Regenerative energiequellen: Praktikum, Springer - Verlag, Berlin (1997).

[10] F.S. Tymvios, C.P. Jacovides and C. Scouteli, "Comparative study of Angstrom's and artificial neural networks methodologies", Solar Energy, Vol. 78, No. 6, pp. 752-762, 2005.

[11] M. Salmi, M. Chegaar and P. Mialhe, "Modèles d'estimation de l'irradiation solaire globale au sol", Revue Internationale d'Heliotechnique Energie \& Environment, No. 35, pp. 19-24, 2007.

[12] R. Sorichetti and O. Perpinán, "PV solar tracking systems analysis”, in Proc. EUPVSEC'2007, Milano, pp. 246-252.

[13] L. Narvarte and E. Lorenzo, "Tracking gains and ground cover ratio”, in Proc. EUPVSEC'2007, Milano, pp. 3153-3156.

[14] K. Karimov, M. Saqib and P. Akhter, "A simple PV tracking system", Solar Energy - Materials and Solar Cells, Vol. 87, No. 1-4, pp. 49-59, 2005.

[15] F. Rubio, M. Ortega and F. Gordillo, "Application of new control strategy for sun tracking", Energy Conversion and Management, Vol. 48, No. 7, pp. 2174-2184, 2007.

[16] J.A. Chojnacki, "Application of fuzzy logic neural network controllers in photovoltaic systems", in Proc. EUPVSEC'2005, Barcelona, pp. 2269-2272.

[17] M. Comşiţ and I. Vişa, "Design of the linkages-type tracking mechanisms by using MBS method", in Proc. IFToMM'2007, Besançon, pp. 582-587.

[18] D. Diaconescu and I. Vişa, "Analysis of the sunearth angles used in the design of the solar collectors trackers", Bulletin of the Transilvania University of Braşov, Vol. 13, No. 48, pp. 99-104, 2006. 